\title{
中 国杉堂
}

\section{充液腔体旋转运动的稳定性理论}

\author{
朱如曾 \\ (中国科学院力学研究所, 北京)
}

\begin{abstract}
摘
要

本文对于具有一个定点, 并充满粘性液体的腔, 在重力场中绕惯性主轴的平衡旋 转运动, 采用 Ляпунов-Movchan 直接法 ${ }^{[1]}$, 就尽可能多样的 Movchan 距离, 推广了 Моисеев 和 Румянцев 的稳定性定理 ${ }^{[2,3]}$, 并对尽可能全佰的腔参数 (包括临界情况 在内)给出了具体的稳定性结论.
\end{abstract}

\section{一、引 言}

研究充枮性液体的腔体旋转运动的稳定性问题，有线性化近似方法和 Ляпунов-Movchan 直接法 ${ }^{[1]}$ (将能量函数取为 Ляпунов 函数时又称为能量法). 两者都获得不少结果 ${ }^{[2-8]}$. 本文 讨论后一种方法, 它可以不带任何近似. 在此方法下, Монсеев 和 Румяцев 已对若干 Movchan 距离获得关于稳定性、渐近稳定性和不稳定性的普遍定理 ${ }^{[2,3]}$, 并且也适合于自引力液体 块. 对于稳定已获得了一些具体判据 ${ }^{[2,3]}$, 而对于渐近稳定和不稳定,具体判据获得较少, 因为 这需要仔细地分析平衡转动态是否孤立.

文献 $[2,3]$ 的一般性定理是对确定的 Movchan 距离而制定的,特别是, 定埋中的“不稳定” 是指本文的 $\rho_{\gamma_{13}}, \rho_{\gamma_{23}}, \omega_{1}, \omega_{2}$ 和 $T_{r}$ (液体关于某个特定参照系的动能)中至少一个不稳定，而j 没有给出更细致的定理.

为了统一而简沾地就尽可能多样的 Movchan 距离, 对尽可能全面的腔参数情况, 给出具 体的稳定性(包括稳定、渐近稳定和不稳定)结论, 必须从 Ляпyнов-Movihan 的有关定理出发, 把文献 $[2,3]$ 的一般性定理加以推广, 使之适合于尽可能多样的 Movchan 距离, 然后以推论的 形式给出各种腔参数情况(包括临界情况)下的具体结论.

\section{二、问题的表述}

设具有定点 $O$ 的充满粘性液体 (密度为 $\rho_{1}$, 动力粘滞系数为 $\mu$ ) 的腔体, 在重力场中以竖 直问上的角速度 $\Omega$ 绕一惯性主轴 $Z$ (相应的主转动惯量为 $C$ ) 作平衡旋转运动, 并设壳液总休 的质量为 $M$, 重心位于 $Z$ 轴上, 与 $o$ 的距离为 $l$, 㙅转动惯量分别为 $A, B, C$. 现在引进三个 直角右旋标架: 
(1) 固定标架 $\left\{0, \boldsymbol{i}_{1}^{n}, \boldsymbol{i}_{2}^{0}, \boldsymbol{i}_{3}\right\} ， \boldsymbol{i}_{3}$ 沿竖直方向向上.

(2) 平衡运动标架 $\left\{0, i_{1}, i_{2}, i_{3}\right\} ， i_{3}$ 始终与 $i_{3}^{0}$ 重合, 此系相对固定标架以匀角速度 $Q i_{3}^{0}$ 旋 转.

（3）腔体固联标架 $\left\{0, \boldsymbol{i}_{1}^{\prime}, \boldsymbol{i}_{2}^{\prime}, \boldsymbol{i}_{3}^{\prime}\right\} ， \boldsymbol{i}_{1}^{\prime} 、 \boldsymbol{i}_{2}^{\prime}$ 和 $\boldsymbol{i}_{2}^{\prime}$ 取为壳液总体的三个上方向，对未扰动态， $\boldsymbol{i}_{3}$ 沿 $\boldsymbol{i}_{3}^{0}$.

基矢之间满足如下关系

$$
\boldsymbol{i}_{i}^{\prime}=\sum_{k=1}^{3} \gamma_{i k} \boldsymbol{i}_{k} \quad(j=1,2,3) .
$$

记 $\boldsymbol{i}_{1}$ 与 $\boldsymbol{i}_{1}$ 间夹角为 $\varphi(0 \leqslant \varphi<2 \pi)$ ，标架 (3) 相对于标架 (2) 的角速度在标架 (2) 上的投 影为 $\left(\omega_{1}, \omega_{2}, \omega_{3}\right)$.

现在在平衡运动标架中观察. 当规定了 $\boldsymbol{r}_{33}$ 的正负号后， $\left(\boldsymbol{r}_{13}, \boldsymbol{r}_{23}, p\right)$ 才能确定 $\left(\boldsymbol{i}_{1}^{\prime}, \boldsymbol{i}_{2}^{\prime}\right.$, $\left.\boldsymbol{i}_{3}^{\prime}\right)$ 的取向,所以系统的态空间 $\mathscr{A}$ 由元素 $P=\left(\boldsymbol{r}_{13}, \boldsymbol{r}_{23}, \varphi, \omega_{1}, \omega_{2}, \omega_{3}, \boldsymbol{v}(\boldsymbol{r}), \pm\right)$ 的全体构 成. 其中各有关量必须满足 $0<\varphi \leqslant 2 \pi, 0 \leqslant r_{13}^{2}+r_{23}^{2}<1, \boldsymbol{v}\left(\boldsymbol{r}\right.$ ) (分量是 $v_{1}, v_{2}, v_{3}$ ) 是 满足不可压条件及在腔内壁上无相对滑动条件的二次连续可微函数, \pm 号表示 $r_{33}$ 的正负性. 我们把 $r_{33}$ 取正、负号的全体元素的集合分別记为 $\mathscr{A}_{+}$和 $\mathscr{A}_{-}$, 初态为 $p_{0}, t$ 时刻的态 $p$ 表 示为 $p=p\left(t, p_{0}\right)$. 我们称这样定义的系统为 SBLC 系统. 我们关心的未扰动平衡转动态 足

$$
p^{(e)}=(0,0,0,0,0,0, \boldsymbol{v}(\mathbf{r}) \equiv 0,+) .
$$

按规定初始扰动态 $p_{0}$ 一定位于 $\mathscr{A}_{+}$中, 其 Movchan 距离 $\rho_{0}$ 是

$$
\rho_{0}=\operatorname{Max}\left(\left|\boldsymbol{r}_{13}\right|,\left|\boldsymbol{r}_{23}\right|,|\varphi|,\left|\omega_{1}\right|,\left|\omega_{2}\right|,\left|\omega_{3}\right|,|\boldsymbol{v}(\boldsymbol{r})|\right) \text {. }
$$

我们将考察系统关于如下 Movchan 距离在 Movchan 意义下的稳定性:

$$
\begin{gathered}
\rho_{r_{13}}=\left|\boldsymbol{\gamma}_{13}\right|, \rho_{r_{23}}=\left|\boldsymbol{r}_{23}\right|, \rho_{\varphi}=|\varphi|, \rho_{\omega_{1}}=\left|\omega_{1}\right|, \rho_{\omega_{2}}=\left|\omega_{2}\right|, \\
\rho_{\omega_{3}}=\left|\omega_{3}\right|, \rho_{v}=\iiint_{\tau} \boldsymbol{v}^{2} d \tau, \rho_{v_{r}}=\iiint_{\tau} \boldsymbol{v}_{r}^{2} d \tau .
\end{gathered}
$$

这里 $\tau$ 是液体所占的空间, $\boldsymbol{v}_{r}$ 是液体相对于腔壳的速度分布. 在某些情况下, 我们也讨论

$$
\begin{gathered}
\rho^{\prime}=\left(\rho_{\omega_{1}}^{2}+\rho_{\omega_{2}}^{2}+\rho_{\omega_{3}}^{2}+\rho_{r_{13}}^{2}+\rho_{r_{23}}^{2}\right)^{1 / 2}, \quad \rho^{\prime \prime}=\left(\rho_{\omega_{3}}^{2}+\rho_{r_{13}}^{2}+\rho_{r_{23}}^{2}\right)^{1 / 2}, \\
\rho^{\prime \prime \prime}=\left(\rho_{\omega_{1}}^{2}+\rho_{\omega_{2}}^{2}+\rho_{\omega_{3}}^{2}+\rho_{r_{23}}^{2}\right)^{1 / 2} .
\end{gathered}
$$

所谓 Movchan 意义下 $p^{(e)}$ 关于某 $\rho$ 是“稳定的”, 是指对于任意给定的 $\varepsilon>0$, 找到 $\delta>$ 0 , 只要初始扰动态 $p_{0}$ 成立 $\rho_{0}\left(p_{0}\right)<\delta$, 则对一切 $t>0$, 使 $\rho\left(p\left(t, p_{0}\right)\right)<\varepsilon$, 否则是 $\rho$ 不稳定.

虽然我们只对系统分析上述几个 Movchan 距离的稳定性, 但是通过如下的引理 1 和引理 2 , 可以轻而易举地引出更多的稳定性结论.

引理 1. 系统关于 $\rho_{a}$ 和 $\rho_{b}$ 是 (渐近)稳定的, 等价于系统关于 $\rho=\sqrt{k_{a} \rho_{a}^{2}+k_{b} \rho_{b}^{2}}$ （渐近） 稳定. $k_{a}$ 和 $k_{b}$ 是两个任意的正常数.

引理 2. 系统关于 $\rho=\sqrt{k_{a} \rho_{a}^{2}+k_{b} \rho_{b}^{2}}$ 不稳定，等价于系统至少关于 $\rho_{a}$ 及 $\rho_{b}$ 中之一不稳 定. 


\section{三、SBLC 系统的稳定性定理}

在标架 (1) 中观察, 系统的角动量的 $i_{3}$ 分量 $L_{3}$ 守恒, 显然成立定理 1 .

定理 1. SBLC 系统的 $p^{(e)}$ 关于 $\rho_{\omega_{3}}$ 和 $\rho_{\boldsymbol{v}}$ 不渐近稳定, 关于 $\rho_{\boldsymbol{q}}$ 不稳定.

为了进一步讨论, 我们定义 $p^{(\rho)}$ 的 $\rho_{0}, \rho_{r}, \rho_{k}$ 及 $\rho$ 邻域

$$
\begin{aligned}
& N_{\varepsilon}\left(\rho_{0}\right)=\left\{p \mid 0<\rho_{0}(p)<\varepsilon, p \in \mathscr{A}_{+}\right\} ; \\
& N_{\varepsilon}\left(\rho_{\gamma}\right)=\left\{p \mid 0<\rho_{\gamma}(p)<\varepsilon, p \in \mathscr{O}_{+}\right\}, \quad \gamma \text { 取 } \gamma_{13}, \gamma_{23} \text {; } \\
& N_{k}\left(\rho_{k}\right)=\left\{p \mid 0<\rho_{k}(p)<\varepsilon, p \in . \sigma_{+}\right\}, \quad k \text { 可取 } \omega_{1}, \omega_{2}, \omega_{2}, \boldsymbol{v}, \boldsymbol{v}_{r} \text {; } \\
& N_{\varepsilon}(p)=\{p \mid 0<\rho(p)<\varepsilon, p \in \mathscr{A}+\}, \quad \rho \text { 取 } \rho^{\prime}, \rho^{\prime \prime}, \rho^{\prime \prime \prime} \text {. }
\end{aligned}
$$

站在标架 (2) 中观察, 根据熟知的机械能耗散率 $\phi$ 的公式 ${ }^{[9]}$

$$
\phi=\frac{1}{2} \iiint_{i} \sum_{i, j=1}^{3} \mu\left(\frac{\partial v_{i}}{\partial x_{i}}+\frac{\partial v_{1}}{\partial x_{i}}\right)^{2} d \tau,
$$

直接得

$$
\frac{d V}{d t}=-\phi \leqslant 0
$$

式中

$$
\begin{aligned}
& V=E+L_{i} \quad\left(j=\left\{\begin{array}{lll}
1 & \text { 当 } p \in \mathscr{A}_{+} \\
2 & \text { 当 } p \in \mathscr{A}_{-}
\end{array}\right),\right. \\
& E=\frac{1}{2} \omega \cdot \mathbf{J} \cdot \boldsymbol{\omega}+\frac{1}{2} \iiint_{\tau} \rho_{1} \boldsymbol{v}^{2} d \tau \\
& L_{1}=\left(\sqrt{1-\left(\gamma_{13}^{2}+\gamma_{23}^{2}\right)}-1\right) M g h+\frac{Q^{2}}{2}\left[\gamma_{13}^{2}(C-A)+\gamma_{23}^{2}(C-B)\right], \\
& L_{2}=-\left(\sqrt{1-\left(\gamma_{13}^{2}+\gamma_{23}^{2}\right)}+1\right) M g h+\frac{Q^{2}}{2}\left[\gamma_{13}^{2}(C-A)+\gamma_{23}^{2}(C-B)\right] \text {, }
\end{aligned}
$$

式中 $\mathbf{J}$ 是壳体绕 $o$ 点的转动惯量张量，(4) 式右边两项分别是壳体动能和液体动能，(5) 式和 (6) 式右边二项分别是重力势能和惯性离心力势能, $h$ 是在 $p^{(e)}$ 态下西液总体的重心以 $o$ 为基 准之高度.

引理 3. 对 SBLC 系统的任一初态 $p_{0}$, 必存在某一 $\phi \equiv 0$ 的轨道 $\mathscr{L}\left(p_{0}\right)$, 它是 $p\left(t, p_{0}\right)$ 按 $\rho_{\nu}^{\prime}, \rho_{v \gamma}^{\prime}, \rho_{\omega_{1}}^{\prime}, \rho_{\omega_{2}}^{\prime}, \rho_{\omega_{3}}^{\prime}, \rho_{Y_{13}}^{\prime}$ 和 $\rho_{r_{23}}^{\prime}$ 的极限点的全体(各 $\rho^{\prime}$ 距离均是 Movchan 意义下的,具体 意义明显, 故从略).

这一引理是以往所有关于充粘性液体腔渐近稳定及不稳定定理的基础 ${ }^{[2]}$.

定理 2. 对于 SBLC 系统，在 $p^{(e)}$ 的某邻域 $N_{\varepsilon}\left(\rho_{\gamma_{13}}\right) \cap N_{\varepsilon}\left(\rho_{r_{23}}\right)$ 内，如果 $(1) L_{1}$ 关于两 个 $\rho_{r}$ 正定(或 $L_{1}$ 和 $L_{2}$ 关于某个 $\rho_{\gamma}$ 正定), 则 $p^{(e)}$ 关于两个 $\rho_{r}$ (或关于该 $\rho_{\gamma}$ ) 稳定; 此外, 如果还满足 (2) 在 $N_{\varepsilon}\left(\rho_{\gamma}\right)$ 内没有 $\phi \equiv 0$ 的整条轨道,则 $p^{(e)}$ 关于该 $\rho_{r}$ 渐近稳定.

证. 由(4)式知, $E \geqslant 0$, 又由已知条件 (1), 在 $N_{\varepsilon}\left(\rho_{r_{13}}\right) \cap N_{\varepsilon}\left(\rho_{\tau_{23}}\right)$ 内, $V$ 关于两个 $\rho_{\gamma}$ (或 关于某个 $\rho_{r}$ ) 正定; $V$ 显然关于 $\rho_{0}$ 连续（已包含 $V\left(p^{(e)}\right)=0$ 在内 ${ }^{[1]}$ ); 由 (2) 式知 $V$ 非增. 根 
据 Movchan 的定理 $(5.1)^{[11}$ 得" $p^{(e)}$ 关于两个 $\rho_{\gamma}$ (或关于该 $\rho_{r}$ ) 稳定. 根据引理 3 及条作 2 , $p^{(e)}$ 关于 $\rho_{r}$ 渐近稳定。证毕.

定理 3. 对于 SBLC 系统, 如果 (1) 在 $p^{(c)}$ 的一个邻域 $N_{\varepsilon}\left(\rho_{r_{13}}\right) \cap N_{\varepsilon}\left(\rho_{r_{23}}\right)$ 内, $L_{1}$ 关于 $=$ $\rho_{r_{13}}$ 和 $\rho_{r_{23}}$ 都正定, 则 $p^{(e)}$ 关于所有的 $\rho_{k}$ 稳定; 如果还满足 (2) 对某一 $\rho_{k}$, 在 $N_{\dot{\varepsilon}}\left(\rho_{k}\right)$ 中 没有 $\phi \equiv 0$ 的整条轨道, 则关于该 $\rho_{k}$ 渐近稳定.

证. 由 (4) 式知, $E$ 关于除 $\rho_{v_{\gamma}}$ 外的所有 $\rho_{k}$ 正定,与定理 2 类似可证, $p^{(e)}$ 关于所有的 $\rho_{k}$ (除 $\rho_{\boldsymbol{y}}$ 外)稳定. 由于 $\boldsymbol{v}_{\boldsymbol{\gamma}}=\boldsymbol{v}-\boldsymbol{\omega} \times \boldsymbol{\gamma}$, 得 $\boldsymbol{v}_{\gamma}^{2} \leqslant 2\left(\boldsymbol{v}^{2}+R^{2} \boldsymbol{\omega}^{2}\right)$ ( $R$ 是腔的最大尺度), 于 是 $\rho_{\boldsymbol{v} \gamma} \leqslant 2\left(\rho_{\nu}+R^{2} \tau \boldsymbol{\omega}^{2}\right)$. 所以 $p^{(\boldsymbol{\rho})}$ 关于 $\rho_{\boldsymbol{v} \gamma}$ 也稳定. 渐近稳定的证明与定理 2 同.

同样可证定理 4 .

定理 4. 对 SBLC 系统, 如果 $L_{1}$ 和 $L_{2}$ 是常正的, 即 $L_{1} \geqslant 0, L_{2} \geqslant 0$, 则 $p^{(e)}$ 关于所有 的 $\rho_{k}$ 稳定; 此外, 如果对某 $\rho_{k}$, 在 $N_{\varepsilon}\left(\rho_{k}\right)$ 内无 $\phi \equiv 0$ 的整条轨道, 则 $p^{(e)}$ 关于该 $\rho_{k}$ 渐近稳 定.

定理 5. 对于 SBLC 系统, 如果 (1) $L_{1}$ 在 $p^{(e)}$ 的任意小 $\rho_{0}$ 邻域 $N_{\delta}\left(\rho_{0}\right)$ 中不常正 (更 不正定)，(2) 存在 $p^{(e)}$ 的某一 $\rho_{\gamma}$ 邻域 $N_{\varepsilon}\left(\rho_{\gamma}\right)$, 在其中 $V<0$ 的区域内并不含有 $\phi \equiv 0$ 的 整条轨道,则 $p^{(e)}$ 关于该 $\rho_{\gamma}$ 不稳定.

证. 由条件 (1), 显然, 在任意小的邻域 $N_{\delta}\left(\rho_{0}\right)$ 中都能找到一点 $p_{0}$, 使 $L_{1}\left(p_{0}\right)<0$, 并 且 $E\left(p_{0}\right)=0$, 故 $V\left(p_{0}\right)<0$. 由 (2) 式知, $V(t)$ 非增, 且显然有界, 故有极限 $V^{*} \leqslant V\left(p_{0}\right)$, 所以在引理 3 所决定的 $\mathscr{L}\left(p_{0}\right)$ 上有 $V=V^{*} \leqslant V\left(p_{0}\right)<0$. 由条件 (2), $\mathscr{L}\left(p_{0}\right) \bar{e} N_{*}\left(\rho_{r}\right)$ $\cup p^{(e)}$. 故 $p^{(e)}$ 对该 $\rho_{\gamma}$ 不稳定.

同样可证定理 6 .

定理 6. 对于 SBLC 系统, 如果 $L_{1}$ 在 $p^{(e)}$ 的任意小 $\rho_{0}$ 邻域 $N_{\delta}\left(\rho_{0}\right)$ 中不常正（更不正 定). 并且存在 $p^{(e)}$ 的某 $\rho_{k}$ 邻域 $N_{\varepsilon}\left(\rho_{k}\right)$ (或其他 $\rho$ 邻域), 在其中 $V<0$ 的区域内, 并不含 有 $\phi \equiv 0$ 的整条轨道,则 $p^{(e)}$ 关于该 $\rho_{k}$ 不稳定(或关于 $\rho$ 不稳定).

上述定理 2 到定理 6 是 Моисеев 和 Румянцев 的定理（文献 [2]第 4 章 §3) 在 SBLC 系统中对多种 Movchan 距离情况的推广.

\section{四、稳定性结 论}

推论 1. 对满足

$$
Q^{2}(C-A)-M g h>0, \quad \text { 且 } \quad Q^{2}(C-B)-M g h>0
$$

的 SBLC 系统, (1) 若 $h \neq 0$, 则 $p^{(e)}$ 关于 $\rho_{\gamma_{13}}, \rho_{\gamma_{23}}, \rho_{\omega_{1}} 、 \rho_{\omega_{2} 2}$ 和 $\rho_{\nu_{\gamma}}$ 渐近稳定, 关于 $\rho_{v}$ 和 $\rho_{\omega_{3}}$ 稳定而不渐近稳定; (2) 若 $h=0$, 则 $p^{(e)}$ 关于 $\rho_{v_{7}}$ 渐近稳定, 关于 $\rho_{\gamma_{13}}, \rho_{\gamma_{23}}, \rho_{\omega_{1}}, \rho_{\omega_{1}}, \rho_{\omega_{3}}$ 利 1 $\rho_{\boldsymbol{v}}$ 稳定而不渐近稳定.

证. 由 (5) 式和 (7) 式可知, 存在 $N_{\varepsilon}\left(\rho_{\gamma_{13}}\right)$ 和 $N_{\varepsilon}\left(\rho_{\gamma_{23}}\right)$, 使在 $N_{\varepsilon}\left(\rho_{\gamma_{13}}\right) \cap N_{\varepsilon}\left(\rho_{r_{23}}\right)$ 中, $L_{1}$ 关于 $\rho_{r_{13}}$ 和 $\rho_{r_{33}}$ 正定, 由定理 2 和 3 得, 本推论的所有有关距离的稳定性. 下面证渐近稳定 性. 根据引理 3 , 扰动轨道 $p\left(t, p_{0}\right)$ 必趋向某 $\phi \equiv 0$ 的轨道 $\mathscr{L}\left(p_{0}\right)$.

1) Movchan 定理 5.1 本来要求任 $\propto$ 中, $V$ 关于 $\rho_{\gamma}$ 正定, 而此处只在 $N_{\varepsilon}\left(\rho_{r_{13}}\right) \cap N_{\varepsilon}\left(\rho_{r_{23}}\right)$ 内正定, 但经简单推理不 难明白, Movchan 定理的结论仍适用. 
1. 当 $h \neq 0$, 从附冰 $A$ 表 2 中查得 $\phi \equiv 0$ 的轨道集有如下三类：（1） $\theta=\pi, W$ 任意. 圆 此 $\gamma_{33}=-1$. 根据 $p^{(e)}$ 关于 $\rho_{r_{13}}$ 和 $\rho_{r_{23}}$ 的稳定性将其排除.

(2) $\cos \theta=\frac{M g l}{W^{2}(C-A)}$ 或 $\cos \theta=\frac{M g l}{W^{2}(C-B)}$. 因为 $p^{(e)}$ 点与 $\theta=0, W=Q$ 对应, 根据 (7) 式, $p^{(e)}$ 点不满足这两 式，所以在 $p^{(e)}$ 点周围存在区域 $N_{\varepsilon}\left(\rho_{r_{1}}\right) \cap N_{\varepsilon}\left(\rho_{r_{13}}\right) \cap N_{\varepsilon}\left(\rho_{w_{1}}\right)$ ，其中不含有这二种轨道．根据 已证明的稳定性将其排除．（3） $\theta=0 ， W$ 任意. 其中必含有 $\mathscr{L}\left(p_{0}\right)$ ，这表明 $p^{(e)}$ 关于 $\rho_{r_{13},}, \rho_{r_{23}}, \rho_{\omega_{1}}, \rho_{\omega_{2}}$ 和 $\rho_{v \boldsymbol{y}}$ 渐近稳定; 由定理 1 , 关于 $\rho_{w_{3}}$ 和 $\rho_{v}$ 不渐近稳定.

2. 当 $h=0$ 时, 从附录 $A$ 表 1 中可知, $\phi \equiv 0$ 的轨道中有 $\theta=0, W$ 任意这一种. 显 然, 在 $p^{(e)}$ 的任意 $\rho_{v}$ 邻域内都存在完整的, 与 $p^{(c)}$ 保持有限距离 $\rho_{r_{13}}, \rho_{r_{23}}, \rho_{\omega_{1}}, \rho_{w_{2}}, \rho_{w 3}$ 和 $\rho_{v}$ 的 这种轨道, 所以 $p^{(e)}$ 关于 $\rho_{r_{13}}, \rho_{r_{23}}, \rho_{\omega_{1}}, \rho_{\omega_{2}}, \rho_{w_{3}}$ 和 $\rho_{v}$ 不渐近稳定. 但白引理 $3, p^{(e)}$ 关于 $\rho_{v r}$ 是 渐近稳定的. 证毕.

推论 2. 对满是

$$
Q^{2}(C-A)-M g h<0 \text {, 且 } Q^{2}(C-B)-M g h \neq 0
$$

的 SBLC 系统, (1) 若 $h=0$, 则 $p^{(e)}$ 关于 $\rho^{\prime}$ 不稳定, (2) 若 $h \neq 0$, 则 $p^{(e)}$ 关于 $\rho^{\prime \prime}$ 不稳 定.

证.（5）式可写为

$$
L_{1}=\frac{1}{2}\left[\Omega^{3}(C-A)-M g h\right] \gamma_{13}^{2}+\frac{1}{2}\left[Q^{2}(C-B)-M g h\right] \gamma_{23}^{2}+\cdots \cdot
$$

认 (8) 式和, $L_{1}$ 在 $p^{(e)}$ 的任意小 $\rho_{0}$ 邻域内不常正(也不正定).

当 $l=0$ 时, (8) 式决定 $A \neq C$, 且 $B \neq C$. 利用附录 $B$ 中引理 4 和 5 得知, 附录 $A$ 表 1 中 $l=0, A \neq C, B \neq C, \phi \equiv 0$ 的全部轨道, 要么 $V \geqslant 0$, 要么与 $p^{(e)}$ 有一有限的 $\rho^{\prime}$ 距离. 由定理 6 , 结论 (1) 得证. 对 $l \neq 0$ 的情况, 从表 2 中查得, $\phi \equiv 0$ 的轨道有如下四 类： $1 . \theta=0, W$ 任意. 因此有 $\gamma_{13}=\gamma_{23}=0, \omega_{3}$ 任意,于是 $V=\frac{1}{2} \omega_{3}^{2} C \geqslant 0.2 . \theta=\pi$, $W$ 任意. 因此 $r_{33}=-1$, 必在某时刻达到 $r_{33}=0$, 即 $r_{13}^{2}+r_{23}^{2}=1.3 . \cos \theta=\frac{M g l}{W^{2}(C-A)}$, 按 (8) 式, $p^{(e)}$ 点不满足此式, 所以在 $p^{(e)}$ 点周围存在一个 $\rho^{\prime \prime}$ 邻域 $N_{\varepsilon_{1}}\left(\rho^{\prime \prime}\right)$, 其中各点均不满 足上式. 4. $\cos \theta=\frac{M g l}{W^{2}(C-B)}$, 与情况 3一样. 根据定理6, 结论 (2) 成立. 证毕.

推论 3. 对满足条件

$$
Q^{2}(C-A)-M g h=0, \quad \text { 且 } \quad Q^{2}(C-B)-M g h \neq 0
$$

的系统

1. 当 $h=0$ (必定 $A=C \neq B$ )

(a) 若 $C>B$, 则 $p^{(e)}$ 关于 $\rho_{v}, \rho_{\gamma: 3}, \rho_{\omega_{1}}, \rho_{\omega_{2}}$ 和 $\rho_{\omega_{3}}$ 稳定但不渐近稳定, 关于 $\rho_{v_{\gamma}}$ 渐近稳 定. (b) 若 $C<B$, 则 $p^{(e)}$ 关于 $\rho^{\prime \prime \prime}$ 不稳定.

2. 若 $h>0$ (必定 $A \neq B, A \neq C$ ), 且 $4 A \geqslant 3 C$, 则 $p^{(e)}$ 关于 $\rho^{\prime \prime}$ 不稳定.

3. 当 $h<0$ (必定 $A \neq B, A \neq C$ )

(a) 若 $Q^{\prime}(C-B)-M g h>0$, 则 $p^{(e)}$ 关于 $\rho_{r_{13}}, \rho_{\omega_{3}}$ 和 $\rho_{v}$ 稳定而不渐近稳定, 关于 $\rho_{\omega_{1}}$, 
$\rho_{\text {lu: }}, \rho_{\gamma_{23}}$ 和 $\rho_{v \gamma}$ 渐近稳定。 (b) 若 $Q^{2}(C-B)-M g<0$ ：且 $4 . A \geqslant 3 C$, 则 $p^{(c)}$ 关于 $\rho^{\prime \prime}$ 不 稳定.

证，当 (9) 式成立时,从（5）式得

$$
\begin{aligned}
L_{1}= & \frac{1}{2}\left[\Omega^{2}(C-B)-M g h\right] \gamma_{23}^{2}-\frac{1}{8}\left(r_{13}^{2}+\gamma_{23}^{2}\right) M g h \\
& -\frac{1}{16}\left(\gamma_{13}^{2}+r_{23}^{2}\right)^{3} M g h+\cdots .
\end{aligned}
$$

1. $h=0$, 从 (5) 式和 (6) 式知, $L_{1}=L_{2}=\frac{1}{2} \Omega^{\prime}(C-B) r_{23}^{2}$.

(a) $C>B, L_{1}$ 和 $L_{2}$ 关于 $\rho_{\gamma_{23}}$ 正定, 又有 $L_{1} \geqslant 0, L_{2} \geqslant 0$, 按定理 2 和 4 得, $p^{(e)}$ 关于 $\rho_{\gamma_{23}}$, $\rho_{v}, \rho_{v_{\gamma}}, \rho_{\omega_{1}}, \rho_{\omega_{2}}$ 和 $\rho_{\omega_{3}}$ 稳定. 从附录 $B$ 表 1 的 $A=C \neq B$ 项中查得, 对 $\phi \equiv 0$ 的轨道, $W$ 可以任 意, 因此不渐近稳定 (除 $\rho_{\boldsymbol{v}}$ 外). (b) $C<B, L_{1}$ 关于 $\rho_{0}$ 不常正: $A=C \neq B, l=0, \phi \equiv 0$ 的轨道 有如下两类: 第一类, $\phi=\frac{\pi}{2}\left(\right.$ 或 $\left.\frac{3 \pi}{2}\right), \boldsymbol{W}$ 和 $\theta$ 任意. 由附录 $B$ 的引理 6 得到 $V \geqslant 0$; 第二 类, $\theta=\frac{\pi}{2}, \psi=0, \boldsymbol{W}$ 任意. 由附录 $B$ 的引理 7 得知这些轨道与 $p^{(e)}$ 有有限的 $\rho^{\prime \prime \prime}$ 距离. 按定理 $6, p^{(e)}$ 关于 $\rho^{\prime \prime \prime}$ 不稳定.

2. $h>0$, 且 $4 A \geqslant 3 C$ (必定 $A \neq B, A \neq C$ ).

从 (10) 式可知, $L_{1}$ 在 $p^{(e)}$ 以 $\rho_{0}$ 为距离的无限近处能取负值. 表 2 中查得 $A \neq B$, $A \neq C, l \neq 0, \phi \equiv 0$ 的轨道有四类. 前三类是 $\theta=0, W$ 和 $\psi$ 任意; $\theta=\pi ; \cos \theta=M g l l$ $W^{2}(C-B)$ (若 $B \neq C$ ), $\psi=0($ 或 $\pi$ ). 对这三类的分析与推论 2 中类似. 第四类是 $\cos \theta=M g l / W^{2}(C-A), \psi=\frac{\pi}{2}\left(\right.$ 或 $\left.\frac{3}{2} \pi\right)$. 此时必定 $r_{23}=0$. 令 $\chi=r_{13}^{2}+r_{23}^{2}>0$, 在此轨道上, 利用 $M g h=Q^{2}(C-A)$ 得

$$
V=\Omega^{2}\left(\frac{4 A-3 C}{32} \chi^{2}+\frac{12.1-7 C}{128} \chi^{3}+\cdots\right) .
$$

因为已规定 $4 A \geqslant 3 C$, 所以对这种轨道, 在 $p^{(e)}$ 以 $\chi$ 为距离的附近 $V \geqslant 0$, 按定理 6 , $p^{(e)}$ 关 于 $\rho^{\prime \prime}$ 不稳定.

3. $h<0$ (必定 $A \neq B, A \neq C$ )

(a) $Q^{2}(C-B)-M g h>0$. 从 (10) 式可知, 存在 $\varepsilon>0$, 在 $N_{\varepsilon}\left(\rho_{\gamma_{13}}\right) \cap N_{\varepsilon}\left(\rho_{\gamma_{23}}\right)$ 中, $L_{1}$ 关于 $\rho_{\tau_{23}}$ 和 $\rho_{r_{13}}$ 正定. 按定理 2 和 $3, p^{(e)}$ 关于 $\rho_{\gamma_{13}}, \rho_{r_{23}}, \rho_{\omega_{1}}, \rho_{\omega_{2}}, \rho_{\omega_{3}}, \rho_{v}$ 利 $\rho_{v \gamma}$ 稳定. 由 于已证明的稳定性, $\left\{\mathscr{L}\left(p_{0}\right)\right\}$ 中不可能包括 $\theta=\pi, \cos \theta=M g l / W^{\prime 2}(C-B)($ 若 $B \neq C$ ), 只可能包括 $\theta=0$ 及 $\cos \theta=M g l / W^{2}(C-A)\left(\right.$ 但 $\phi=\frac{\pi}{2}$ 或 $\left.\phi=\frac{3}{2} \pi\right)$. 对后面这二种轨 道, 都有 $\omega_{1}=\omega_{2}=\gamma_{23}=\boldsymbol{v}_{r}=0$, 所以 $p^{(e)}$ 关于 $\rho_{\omega_{1}}, \rho_{\omega_{2}}, \rho_{\gamma_{23}}$ 和 $\rho_{\omega r}$ 渐近稳定. 因为 $p^{(e)}$ 点适 合 $\cos \theta=M g l / W^{2}(C-A)$, 所以不管 $\varepsilon$ 多么小, 我们都可以找到 $p(0)$ 点, 使它满足 $0<\rho_{0}(p(0))<\varepsilon$ 和 $\cos \theta=\frac{M g l}{W^{2}(C-A)}$,于是它永远在这条轨道上. 容易证明, 这轨道与 $p^{(e)}$ 有有限的 $\rho_{r_{13}}, \rho_{\omega,}$ 和 $\rho_{\boldsymbol{v}}$ 距离, 从而 $p^{(c)}$ 关于这些距离不渐近稳定.

(b) $\overline{Q^{3}}(C-B)-M g h<0$, 且 $4 A \geqslant 3 C$. 与第 2 项相似的分析可证 $p^{(e)}$ 关于 $\rho^{\prime \prime}$ 不 
稳定. 证毕.

推论 4. 对满足条件

$$
\Omega^{2}(C-A)-M g h=Q^{2}(C-B)-M g h=0
$$

(必定有 $A=B$ ) 的 SBLC 系统

1. 若 $h<0$, 并且 $A=B \neq C$, 则 $p^{(e)}$ 关于 $\rho_{\omega_{1}}, \rho_{\omega_{1}}, \rho_{u_{\gamma}}$ 渐近稳定, 关于 $\rho_{r_{13}}, \rho_{r_{23}}, \rho_{\omega_{3}}$ 和 $\rho_{\text {。 }}$ 稳定而不渐近稳定. 若 $h<0$, 且 $A=B=C$, 则关于 $\rho_{\gamma_{13}}, \rho_{r_{23}}, \rho_{w_{1}}, \rho_{\omega_{2}}$, 和 $\rho_{v_{y}}$ 渐近稳 定, 关于 $\rho_{\omega_{3}}$ 和 $\rho_{v}$ 稳定而不渐近稳定.

2. 若 $h=0$ (必有 $A=B=C$ ), 则 $p^{(e)}$ 关于 $\rho_{y}, \rho_{\omega_{1}}, \rho_{\omega_{2}}$ 和 $\rho_{\omega_{3}}$ 稳定但不渐近稳定, 关于 $\rho_{\text {*r }}$ 渐近稳定.

3. 若 $h>0$ (必定 $A=B \neq C$ ), 且 $4 A \geqslant 3 C$, 则 $p^{(e)}$ 关于 $\rho "$ 不稳定.

证.

$$
L_{1}=-\frac{1}{8}\left(\gamma_{13}^{2}+\gamma_{23}^{2}\right) M g h-\frac{1}{16}\left(\gamma_{13}^{2}+\gamma_{23}^{2}\right)^{3} M g h+\cdots .
$$

$1 . h<0$, 从 (13) 式知 $L_{1}$ 关于 $\rho_{r_{13}}$ 和 $\rho_{r_{23}}$ 正定. 按定理 2 和 3 得 $p^{(e)}$ 关于有关距离的 稳定性. 若 $A=B \neq C$, 则 $\phi \equiv 0$ 的轨道中包括 $\theta=0$ 及 $\cos \theta=M g l / W^{2}(C-A)(\phi$ 任意), 所以关于 $\rho_{\omega_{1}}, \rho_{\omega_{2}}, \rho_{v_{r}}$ 渐近稳定, 其余距离则不渐近稳定. 若 $A=B=C$, 则 $\phi \equiv 0$ 的 轨道中, 只有 $\theta=0$, 因此关于 $\rho_{\gamma_{13}}, \rho_{\gamma_{23}}, \rho_{\omega_{1}}, \rho_{\omega_{2}}$ 和 $\rho_{\nu \gamma}$ 渐近稳定,关于 $\rho_{\omega_{3}}$ 和 $\rho_{\nu}$ 不渐近稳定.

2. $h=0$. (5) 式和 (6) 式给出 $L_{1}=L_{2}=0$. 按定理 4, $p^{(e)}$ 关于 $\rho_{v}, \rho_{\omega_{1}}, \rho_{\omega_{2}}$ 和 $\rho_{\omega_{3}}$ 稳 定.不渐近稳定是显然的.

3. $h>0$. 此时必定 $A=B \neq C . \quad l \neq 0, C \neq A=B, \phi \equiv 0$ 的轨道有如下三类:

第一类, $\theta=0$ 和第二类 $\theta=\pi$ 的分析同推论 2 的有关部分. 第三类, $\cos \theta=M \mathrm{gl} /$ $W^{2}(C-A)$. 仍令 $\chi=r_{13}^{2}+r_{23}^{2}>0$, 利用 $A=B$ 得 (11) 式. 当 $4 A \geqslant 3 C$ 时, $V \geqslant 0$. 按定理 $6, p^{(e)}$ 关于 $\rho^{\prime \prime}$ 不稳定. 证毕.

\section{附录 A. SBLC 系统 $\phi \equiv 0$ 的轨道}

在固定参考系里观察，利用 $\phi \equiv 0$ 条件是壳液总体以常角速度 $W$ 作整体刚性运动。取固定标架 $(\xi, 亏$ ， $\eta)$ ，使 $\zeta$ 轴与 $W$ 重合. 标架 $\left(i_{1}^{\prime}, i_{2}^{\prime}, i_{3}^{\prime}\right)$ 的方位用尤拉角 $(\theta, \psi, \varphi)$ 表示. 解刚体运动的无拉方程所得 结果示于表 1 和 2.

\begin{tabular}{|c|c|c|}
\hline 条件 & 轨 & $\boldsymbol{W}$ 的方向 \\
\hline \multirow{2}{*}{$A \neq B \neq C$} & (1) $\theta=0$ (或 $\pi), W, \psi$ 任意 & $W$ 与 $i_{3}^{\prime}$ 更台 \\
\hline & (2) $\theta=\frac{\pi}{2}, \psi=\frac{n \pi}{2},(n=0,1,2,3), W$ 任䛓 & $W$ 与 $i_{1}^{\prime}$ 或 $i_{2}^{\prime}$ 重问 \\
\hline \multirow{2}{*}{$A=B \neq C$} & (1) $\theta=0$ (或 $\pi$ ), $\boldsymbol{W}, \psi$ 任意 & $W$ 与 $i_{3}^{\prime}$ 重合 \\
\hline & (2) $\theta=\frac{\pi}{2}, W, \psi$ 任意 & $W$ 与 $i_{3}$ 垂㚗 \\
\hline
\end{tabular}

表 $1 l=0, \phi \equiv 0$ 情况下的运动 
续装 1

\begin{tabular}{|c|c|c|}
\hline 采件 & 轨 & $W$ 的污间 \\
\hline \multirow{2}{*}{$A=c \neq B$} & (1) $\psi=\frac{\pi}{2}\left(\right.$ 或 $\left.\frac{3}{2} \pi\right), \theta, W$ 任意 & $W \perp i_{2}$ \\
\hline & (2) $\theta=\frac{\pi}{2}, \psi=0$ (或 $\pi$ )，W 任意 & $W i_{2}$ \\
\hline \multirow{3}{*}{$B=C \neq A$} & (1) $\theta=0($ 或 $\pi), \boldsymbol{W}, \psi$ 任意 & $\boldsymbol{W}, i_{3}$ \\
\hline & (2) $\psi=0$ (或 $\pi), \theta, W$ 任意 & $\boldsymbol{W}_{\perp i_{1}}$ \\
\hline & (3) $\theta=\frac{\pi}{2}, \psi=\frac{\pi}{2}\left(\right.$ 或 $\left.\frac{3}{2} \pi\right), W$ 任意 & $\boldsymbol{W} i_{1}$ \\
\hline$A=B=\ell$ & $\phi, \theta, \boldsymbol{W}$ 均任意 & $\boldsymbol{W}$ 任意 \\
\hline
\end{tabular}

表 $2 l \neq 0, \phi \equiv 0$ 情治下的运动

\begin{tabular}{|c|c|c|}
\hline 条 件 & 轧 & $\boldsymbol{W}$ 的方向 \\
\hline \multirow{3}{*}{$A \neq B \neq C$} & (1) $\theta=0$ (或 $\pi) ， \psi$ 和 $W$ 任意 & \multirow{10}{*}{$W / i_{3}$} \\
\hline & (3) $\cos \theta=N \mathrm{~g} l / W^{2}(C-B), \psi=0$ (或 $\left.\pi\right)$ & \\
\hline & (3) $\cos \theta=M g l / W^{2}(\dot{C}-A), \psi=\frac{\pi}{2}\left(\right.$ 或 $\left.\frac{3}{2} \pi\right)$ & \\
\hline \multirow{2}{*}{$A=B \neq C$} & (1) $\theta=0$ (或 $\pi), n ， \psi$ 任意 & \\
\hline & (2) $\cos \theta=M / g l / W^{\prime 2}(C-A), \psi$ 任意 & \\
\hline \multirow{2}{*}{$A=C: \neq B$} & (1) $\theta=0($ 㖪 $\pi), \psi, w^{\prime}$ 任意 & \\
\hline & (2) $\cos \theta=M g g l / W^{2}(C-B), \psi=0($ 或 $\pi)$ & \\
\hline \multirow{2}{*}{$B=C \neq A$} & (1) $\theta=0($ 或 $\pi), W, \psi$ 代意 & \\
\hline & (2) $\cos \theta=M_{g} l / I^{\prime 2}(C-A), \quad \psi=\frac{\pi}{2}\left(\right.$ 或 $\left.\frac{3}{2} \pi\right)$ & \\
\hline$A=B=C$ & $\theta=0($ 或 $\pi), W, \psi$ 低意 & \\
\hline
\end{tabular}

\section{附录B. 若 干引理}

引理 4. 对 SBLC 系统, 若 $l=0$, 其 $\phi \equiv 0$ 的轨道中, $\theta=0$ (或 $\pi$ ), $W, \psi$ 任意的那些类, 必有 $V \geqslant 0$.

证. 以 $\theta=0$ 为例. $W / i_{3}^{\prime}$, 令 $i_{3}$ 与 $W$ 夹角为 $\alpha$, 如图 1 所示. 图 1 是在固定参考系 $\left(i_{1}^{0}, i_{2}^{0}, i_{3}^{0}\right)$ 中 画的。 $i_{1}^{\prime}$ 轴绕 $W$ 转动。考虑 $i_{1}^{\prime \prime}, i_{3}$ 积 $i_{3}^{\prime}$ 正好处于局一平面那一瞬间. 此时有

$$
\begin{gathered}
\omega=W-\Omega=-Q \sin \alpha \cdot i_{1}^{\prime}+\left(w^{\prime} \pm \Omega \sin \alpha\right) i_{3}^{\prime}, \\
E=\frac{1}{2} \cdot d Q^{2} \sin ^{2} \alpha+\frac{1}{2} C(\| \pm \Omega \cos \theta)^{2} \geqslant \frac{1}{2} A Q^{2} \sin ^{2} \alpha=\frac{1}{2} A Q^{2}\left(r_{13}^{2}+r_{23}^{2}\right) .
\end{gathered}
$$

此时还有 $r_{23}^{-}=0$, 故 


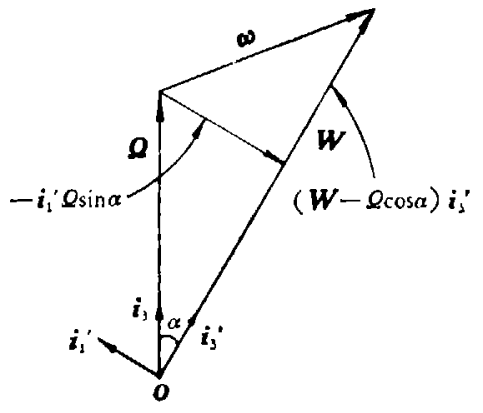

图 1

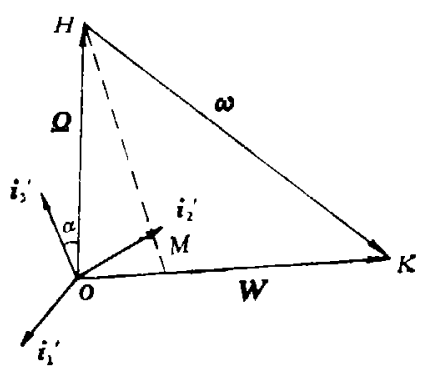

图 2

$$
\begin{gathered}
I_{1}=\frac{\Omega^{2}}{2} \gamma_{13}^{2}(C-.4), \\
V=E+L_{1} \geqslant \frac{Q^{2}}{2} \gamma_{13}^{2} C \geqslant 0 .
\end{gathered}
$$

因为 $\phi \equiv 0$ ，故 $V$ 守恒，所以对整个轨道均有

$$
v \geqslant 0
$$

证毕.

引理 5. 对 $l=0$ 的 SBLC 系统, $\phi \equiv 0$ 的轨道中 $\theta=\frac{\pi}{2}, \phi$ 和 $W$ 任意的那些类, 必与 $p^{(0)}$ 有一有 限的 $\rho^{\prime}$ 距离.

证. $\theta=\frac{\pi}{2}$ 表示 $\boldsymbol{W}_{\perp} \boldsymbol{i}_{3}$. 㹣 2 是在固定参考系里画的.

$$
\omega=W-\Omega, \quad \sum_{i=1}^{3} \omega_{i}^{2}=\omega^{2} .
$$

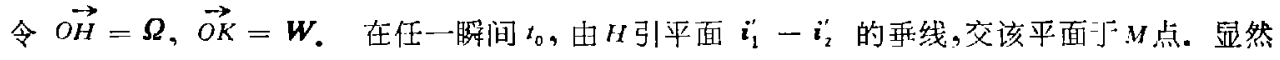

$$
|\omega| \geqslant|H M|=|e \cos \alpha| \text {, }
$$

此处 $\alpha$ 是: $t_{0}$ 时刻 $\Omega$ 与 $i_{3}$ 的夹角. 所以

$$
\begin{gathered}
\sum_{i=1}^{3} \omega_{i}^{2}=\omega^{2} \geqslant Q^{2} \cos ^{2} \alpha=Q^{2} r_{33}^{2}=Q^{2}\left(1-r_{13}^{2}-r_{23}^{2}\right), \\
\rho^{\prime}=\left[r_{13}^{2}+r_{23}^{2}+\sum_{i=1}^{3} \omega_{i}^{2}\right]^{\frac{1}{2}} \geqslant\left[Q^{2}+\left(1-\Omega^{2}\right)\left(r_{13}^{2}+r_{23}^{2}\right)\right]^{\frac{1}{2}} .
\end{gathered}
$$

因为 $0 \leqslant r_{13}^{2}+r_{23}^{2} \leqslant 1$, 所以

证毕.

$$
\rho^{\prime} \geqslant\left[\min \left(\theta^{2}, 1\right)\right]^{\frac{1}{2}}
$$

引理 6. 对 $l=0$, 且 $A=C$ 的 SHLC 系统, $\phi \equiv 0$ 的轨道中, $\psi=\frac{\pi}{2}\left(\right.$ 或 $\left.\frac{3}{2} \pi\right), \theta$ 和 $\boldsymbol{W}$ 任意的那 些类, 必有 $v \geqslant 0$.

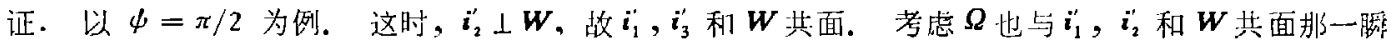
间. $r_{23}=\cos \left(\Omega, i_{2}^{\prime}\right)=0$, 又已知 $t=C$, 由公式 (5) 得 $L_{1}=L_{2}=0$, 所以 $V \geqslant 0$. 由 $v$ 的守恒性, 外 对所有时刻 $V \geqslant 0$. 证毕。

引理 7. 对 $l=0$ 的 SBLC 系统, $\phi \equiv 0$ 的轨道中, $\theta=\pi / 2, \psi=0$, fl $W$ 任意的朋些类型, 与 $p^{(e)}$ 有有限的 $\rho^{\prime \prime \prime}$ 距离. 
证. $\theta=\pi / 2$, 且 $\psi=0$, 表示 $\boldsymbol{W}_{/ / \boldsymbol{i}_{2}^{\prime}}$. 在 $\left(\boldsymbol{i}_{1}^{\prime}, \boldsymbol{i}_{2}, \boldsymbol{i}_{3}\right)$ 上投影得 $\boldsymbol{W}=(0, w, 0), \boldsymbol{\Omega}=\boldsymbol{Q}\left(\boldsymbol{r}_{13}, \boldsymbol{r}_{33}\right.$ ， $r_{33}$. 故

$$
\begin{gathered}
\sum_{i=1}^{3} \omega_{s}^{2}=|W-\Omega|^{2}=Q^{2}\left(r_{13}^{2}+r_{33}^{2}\right)+\left(W-Q r_{23}\right)^{2} \geqslant \Omega^{2}\left(r_{13}^{2}+r_{33}^{2}\right) . \\
\rho^{\prime \prime \prime}=\left[r_{23}^{2}+\sum_{i=1}^{3} \omega_{l}^{2}\right]^{\frac{1}{2}} \geqslant\left[r_{23}^{2}+Q^{2}\left(r_{13}^{2}+r_{33}^{2}\right)\right]^{\frac{1}{2}} \\
=\left[Q^{2}+\left(1-\Omega^{2}\right) r_{23}^{2}\right]^{\frac{1}{2}} \geqslant\left[\min \left(Q^{2}, 1\right)\right]^{\frac{1}{2}} .
\end{gathered}
$$

证毕.

作者感谢谈镐生教授和王照林教授的热情指导和大力帮助.

\section{参考文献}

[1] Movehan, A. A., Appl. Math. Mech. N. Y., 23 (1959), 686.

[2] Monсеев, Н. Н., Румянцев, В. В., Диналика тела : полостями, содержаццили жидкость, М., Изд-во \&Наука», 1965.

[3] 王照林等,现代控制理论基础,国防工业出版社，1981，第九章.

[4] Черноусько, Ф. Л., Двежение твердого тела с колостями, содержащими вязкую экидкость, М., В. Ц. AHCCCP, 1968.

[5] Румянцев, В. В., ПММ., 33(1969).

[6] Соволев, С. Л., ПМТФ, 3(1960), 20-55.

[7] Нилинский, А. Ю.. Темченко, М. Е., ihid., 3(1960), 65-75.

[8] 徐硕昌,中国科兴, 1979,9:85\%。

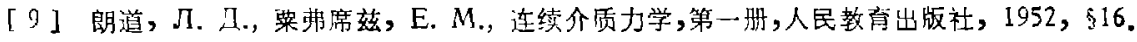

